\title{
Evolução do perfil epidemiológico da aids entre idosos no brasil desde 2009 até 2019
}

\author{
Evolution of the epidemiological profile of aids among the elderly in brazil from 2009 to \\ 2019
}

Evolución del perfil epidemiológico del sida en los ancianos en brasil de 2009 a 2019

João Pedro Moraes Borges ${ }^{1 *}$, Jamilly Gusmão Coelho ${ }^{1}$, Gabriel Cheles Nascimento Matos², Ronald Pinto Costa ${ }^{3}$, Fernanda Gabriele Gordiano Ramos da Silva ${ }^{1}$, Bárbara Souza Fonseca ${ }^{1}$, Aline Benevides Sá Feres ${ }^{1}$, Pedro Fonseca de Vasconselos ${ }^{1}$, Diêgo Andrade de Oliveira ${ }^{1}$, Rosângela Souza Lessa ${ }^{1}$.

\section{RESUMO}

Objetivo: Analisar a prevalência dos casos de AIDS e óbitos nos idosos desde 2009 até 2019 no Brasil. Métodos: Trata-se de uma pesquisa descritiva quantitativa com análise de dados secundários referente ao número de casos de AIDS e óbitos pela AIDS nos indivíduos com 60 anos e mais de acordo com o sexo. Para tanto, foram utilizados as informações do Sistema de Informação e Agravos de Notificação (SINAN), Sistema de Informação sobre Mortalidade (SIM), Sistema de Controle de Exames Laboratoriais (SISCEL) e Sistema de Controle Logístico de Medicamentos (SICLOM) no TABNET e Boletim Epidemiológico de HIV/AIDS 2020. Resultados: Foram notificados 15,672 casos de AIDS entre os idosos do período de 2009 2019 com um pico no ano de 2017, sendo 9,588 do sexo masculino e 6,084 do sexo feminino. Verificou-se a ocorrência de 8,650 óbitos entre homens e 4,257 nas mulheres, uma diferença de, aproximadamente, 50,8\%, somando 12,907 mortes. Conclusão: Conclui-se que os idosos estão sendo mais acometidos pela AIDS com o passar dos anos, dessa forma, é fundamental o desenvolvimento de políticas governamentais voltadas para a educação desses indivíduos e capacitação dos profissionais de saúde acerca da prevenção e abordagem dessa faixa de idade.

Palavras-chave: HIV, AIDS, Idosos, Epidemiologia, Brasil.

\section{ABSTRACT}

Objective: To analyze the prevalence of AIDS cases and deaths in the elderly from 2009 to 2019 in Brazil. Methods: This is a descriptive quantitative research with analysis of secondary data regarding the number of AIDS cases and AIDS deaths in individuals aged 60 years and over, according to gender. For this purpose, information from the Information System and Notifiable Diseases (SINAN), Mortality Information System (SIM), Laboratory Testing Control System (SISCEL) and Drug Logistics Control System (SICLOM) in TABNET and HIV/AIDS Epidemiological Bulletin 2020. Results: 15,672 cases of AIDS were reported among the elderly in the period 2009 - 2019 with a peak in 2017, with 9,588 males and 6,084 females. There were 8,650 deaths among men and 4,257 among women, a difference of approximately $50.8 \%$, totaling 12,907 deaths. Conclusion: It was concluded that the elderly are being more affected by AIDS over the years, thus, it is essential to develop government policies aimed at educating these individuals and training health professionals about the prevention and approach of this age group.

Keywords: HIV, AIDS, Elderly, Epidemiology, Brazil.

\section{RESUMEN}

Objetivo: Analizar la prevalencia de casos y muertes de sida en ancianos de 2009 a 2019 en Brasil. Métodos: Se trata de una investigación cuantitativa descriptiva con análisis de datos secundarios sobre el

\footnotetext{
${ }^{1}$ Faculdade Santo Agostinho (FASA), Vitória da Conquista - BA. *E-mail: joaopedro.moraes@hotmail.com

2 Centro Universitário Aparício Carvalho (FIMCA), Porto Velho - RO.

${ }^{3}$ Centro Universitário São Lucas (UNISL), Porto Velho - RO.
} 
número de casos de sida y defunciones por sida en personas de 60 años y más, según sexo. Para ello, información del Sistema de Información y Enfermedades Notificables (SINAN), Sistema de Información de Mortalidad (SIM), Sistema de Control de Pruebas de Laboratorio (SISCEL) y Sistema de Control Logístico de Medicamentos (SICLOM) en TABNET y Boletín Epidemiológico VIH / SIDA 2020. Resultados: Se notificaron 15.672 casos de sida entre ancianos en el período 2009 - 2019 con un pico en 2017, con 9.588 hombres y 6.084 mujeres. Hubo 8.650 muertes entre hombres y 4.257 entre mujeres, una diferencia de aproximadamente 50,8\%, totalizando 12.907 muertes. Conclusión: Se concluye que los adultos mayores están siendo los más afectados por el SIDA a lo largo de los años, por lo que es fundamental desarrollar políticas gubernamentales orientadas a educar a estos individuos y capacitar a los profesionales de la salud sobre la prevención y abordaje de este grupo de edad.

Palabras clave: VIH, SIDA, Anciano, Epidemiología, Brasil.

\section{INTRODUÇÃO}

A população mundial está vivendo mais e, por isso, envelhecendo, no Brasil, em 1950, a população de idosos era cerca de $4,9 \%$ da população total, em 2060 , espera-se que $32,2 \%$ dos brasileiros faça parte dessa faixa etária (TRAVASSOS GF, et al., 2020). Além disso, em relação à expectativa de vida no Brasil, houve um aumento de 30,8 anos quando comparados os dados de 1940 e 2018 (CRELIER C, 2019). Dessa forma, percebe-se que os avanços tecnológicos e sua aplicabilidade no campo da saúde impactaram no aumento da expectativa de vida e queda da mortalidade da população (GUARNIERI AP, 2008).

Devido ao rápido envelhecimento populacional, surgem novos desafios, como a exposição dos idosos a patologias que, antes, não acometiam essa população de forma significativa, a exemplo das Infecções Sexualmente Transmissíveis (ISTs), com destaque para a infecção pelo Vírus da Imunodeficiência Humana (HIV) e, consequentemente, a Síndrome da Imunodeficiência Adquirida (AIDS) (SOUSA LRM, et al., 2019).

Desde o surgimento dos primeiros casos de AIDS no final da década de 1970, essa patologia tem sido alvo de atenção no mundo inteiro (SILVA IG, et al., 2015). Na atualidade, ela continua sendo importante objeto de investigações sociais devido aos danos que ainda gera a população, como: isolamento devido ao preconceito, efeitos colaterais das medicações nos pacientes em tratamento e até morte (SOUSA LRM, et al., 2019).

Os casos de HIV e AIDS no Brasil crescem desde a identificação do primeiro caso. No entanto, o perfil desse problema de saúde pública sofreu algumas modificações, acima de tudo, em relação às faixas etárias atingidas, o que é demonstrado pela elevação do número de idosos infectados pelo HIV. Em 2009, foram notificados pelo Sistema de Informação de Agravos de Notificação (SINAN) 206 casos de HIV entre os indivíduos idosos, já em 2019, o número cresceu para 1,617, o que representa um aumento de, aproximadamente, $87,3 \%$ (BRASIL, 2020).

A parcela da população com 60 anos ou mais, mesmo com as alterações biopsicossociais advindas do processo de envelhecimento, consegue manter vida ativa, inclusive, sexual, devido aos avanços da saúde e qualidade de vida dos indivíduos em geral. Entretanto, pela falta de políticas públicas específicas, por falta de informação e pela existência de preconceito da sociedade, dos médicos e dos próprios idosos, essa faixa etária acaba sendo infectada pelo HIV e, com o tempo, desenvolvem a AIDS (UCHOA YS, et al., 2016).

Tendo isso em vista, faz-se necessário acompanhar e avaliar o número de casos de HIV e AIDS em idosos de forma detalhada para que a população, os profissionais de saúde e as esferas governamentais tenham conhecimento da dimensão do problema o qual precisa ser enfrentado e planejem estratégias específicas de saúde para os idosos, contribuindo, assim, com o envelhecer saudável e de qualidade (SILVA IG, et al., 2015).

Dessa forma, o presente estudo buscou analisar a evolução do perfil dos casos de AIDS em idosos desde janeiro de 2009 até dezembro de 2019, fazendo um comparativo entre os indivíduos do sexo masculino e feminino com 60 anos e mais, além de destacar o número de óbitos pela doença de acordo com o sexo entre as pessoas inseridas nessa faixa etária. 


\section{MÉTODOS}

Esse trabalho abrange a área de conhecimento das ciências da saúde, é caracterizado como uma pesquisa descritiva e exploratória com abordagem quantitativa que teve como propósito analisar dados secundários referentes à progressão da prevalência de AIDS entre os indivíduos de 60 anos e mais.

A pesquisa avaliou a AIDS no Brasil de acordo com o ano de notificação em indivíduos idosos separados de acordo com o sexo, não sendo levado em consideração quando esse último tópico foi ignorado. Os dados avaliados estão inseridos entre janeiro de 2009 e dezembro de 2019, a fim de perceber a evolução do número de casos de AIDS nos idosos separados de acordo com o sexo. Da mesma forma, foi analisada a prevalência dos óbitos por AIDS nos idosos de forma geral e comparada e estratificada de acordo com o sexo (Figura 1). Para, além disso, não foi levado em consideração o ano de diagnóstico, a raça/ cor, escolaridade e orientação sexual dos sujeitos.

Figura 1 - Fluxograma da população idosa estudada e do período de tempo comparados, em relação ao número de casos e óbitos por AIDS de acordo com o sexo.

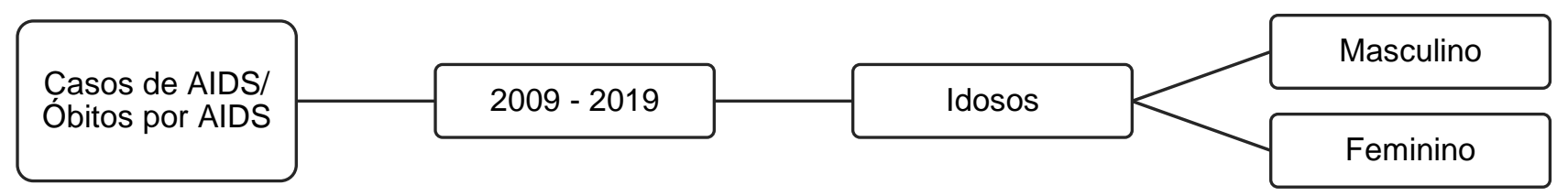

Fonte: Borges JPM, et al., 2021.

Para a obtenção dos números referentes à AIDS nos idosos, foram buscados dados no Sistema de Informação e Agravos de Notificação (SINAN), Sistema de Informação sobre Mortalidade (SIM), Sistema de Controle de Exames Laboratoriais (SISCEL) e Sistema de Controle Logístico de Medicamentos (SICLOM), os quais estão disponíveis no TABNET que foi acessado por meio do site do DATASUS (https://datasus.saude.gov.br/). Para tanto, seguiram-se os subsequentes comandos disponíveis no site: "Acesso à Informação" >> "TABNET" >> "Epidemiológicas e Morbidade" >> "Casos de Aids - Desde 1980 (SINAN)" >> "Aids - desde 1980" >> "Brasil por Região, UF e Município".

Com a finalidade de obter dados referentes aos óbitos por AIDS entre os idosos, foi acessado também o Boletim Epidemiológico de HIV/AIDS 2020 por meio do endereço eletrônico do Ministério da Saúde (2020), seguindo as seguintes coordenadas: "Assuntos" >> "Boletins Epidemiológicos" >> "Por Assunto" >> "AIDS" >> "Boletim Epidemiológico de Aids 2020".

A princípio, com o auxílio do TABNET, foram selecionados os dados a seguir: casos de AIDS em indivíduos com 60 anos e mais entre 2009-2019; casos de AIDS em indivíduos com 60 anos ou mais de acordo com o sexo entre 2009-2019, para esta finalidade, foram seguidos os subsequentes tópicos do TABNET: "Linha (Ano Notificação)" >> "Coluna (Sexo)" >> "Conteúdo (Frequência)" >> "Período (20092019)" >> "Idade detalhada (60-64; 65-69; 70-74; 75-79; 80 anos e mais)". Os dados referentes aos óbitos por AIDS foram coletados Segundo o sexo e faixa etária por ano de óbito (número e coeficiente de mortalidade por 100,000 hab.) entre 2009 e 2019, utilizando o Boletim Epidemiológico de HIV/AIDS 2020, não sendo levados em conta os números relacionados à faixa etária >> "Ignorado".

Após o levantamento realizado, com o intuito de analisar e relacionar os dados, foram construídos gráficos evidenciando a evolução dos casos ao longo do período estudado. No Gráfico 01 foi evidenciada a evolução dos casos de AIDS desde 2009 até 2019, sendo exposto o número de notificações em cada ano 
entre os indivíduos com 60 anos e mais sem distinção de sexo. A diferença entre o acometimento dos idosos de acordo com o sexo de 2009 a 2019 foi expressa no Gráfico 02. Por conseguinte, os óbitos por AIDS entre os sujeitos com 60 anos ou mais de acordo com o sexo no mesmo período foram representados no Gráfico 03.

Posteriormente, foi feita a média do número de casos entre 2009-2019 somando as notificações de todos os anos e dividindo pelo número de anos que foram onze. Em seguida, apontou-se o ano de pico e de maior declínio das notificações nos idosos e a diferença do número de casos notificados nos dois anos. Os casos de AIDS entre os idosos também foram confrontados de acordo com o sexo no espaço de tempo proposto, sendo feito o cálculo de porcentagem da diferença entre o número de homens e mulheres acometidos.

Os óbitos por AIDS acometendo as pessoas a partir de 60 anos foram comparados inicialmente sem distinção dos sexos entre 2009-2019, logo após, foi calculada a média de óbitos no intervalo temporal, também, evidenciado o ano de maior e menor número de casos. Seguidamente, repetiu-se a análise levando em conta o sexo da população e calculou-se a diferença percentual da soma de todos os anos analisados entre homens e mulheres.

Por se tratarem de dados secundários de caráter público, não há exigência de apreciação e aprovação do Comitê de Ética em Pesquisa, entretanto, os dados serão trabalhados com todo rigor, respeitando os preceitos éticos na pesquisa.

\section{RESULTADOS E DISCUSSÃO}

No Brasil, entre os anos de 2009 até 2019, foram registrados 15,672 casos de AIDS na faixa etária de 60 anos e mais, o que gerou uma média de, aproximadamente, 1,425 casos de AIDS por ano (Gráfico 1). Nesse espaço de tempo, houve um pico de notificações entre idosos com 1,619 casos no ano de 2017, ademais, o maior declínio ocorreu em 2010, quando foram contabilizados 1,157 indivíduos, uma diferença de 462 casos entre os dois anos. Ao estratificar o número de casos de acordo com a faixa etária dos idosos, os mais acometidos foram as pessoas entre 60 e 64 anos (Gráfico 2).

Gráfico 1 - Casos de AIDS identificados no Brasil por ano de notificação no período de 2009 a 2019 entre os indivíduos com 60 anos e mais.

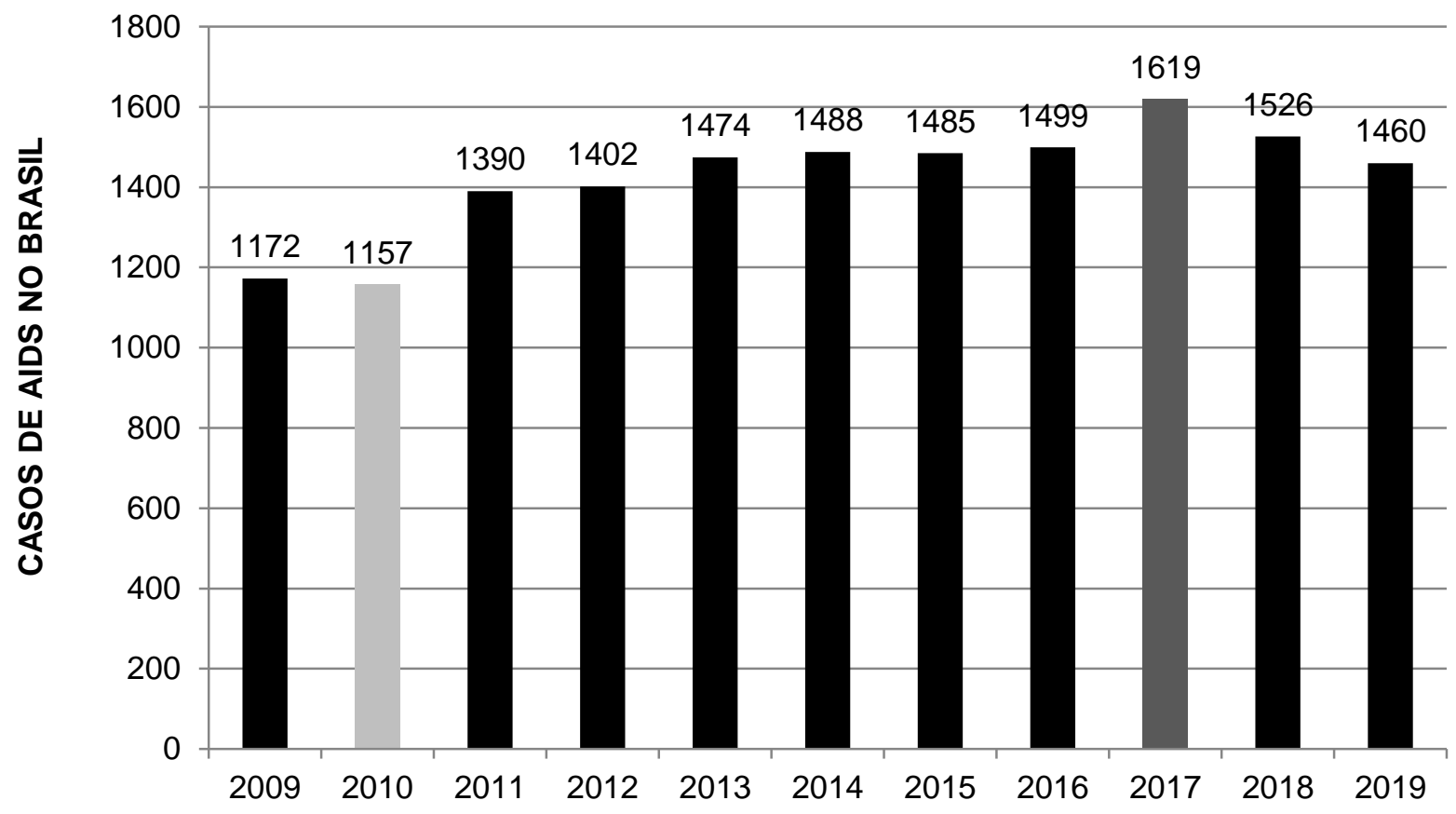

Fonte: Borges JPM, et al., 2021; dados extraídos de TABNET, 2021. 
Gráfico 2 - Casos de AIDS notificados no Brasil de acordo com a faixa etária de idosos em anos no período de 2009 a 2019.

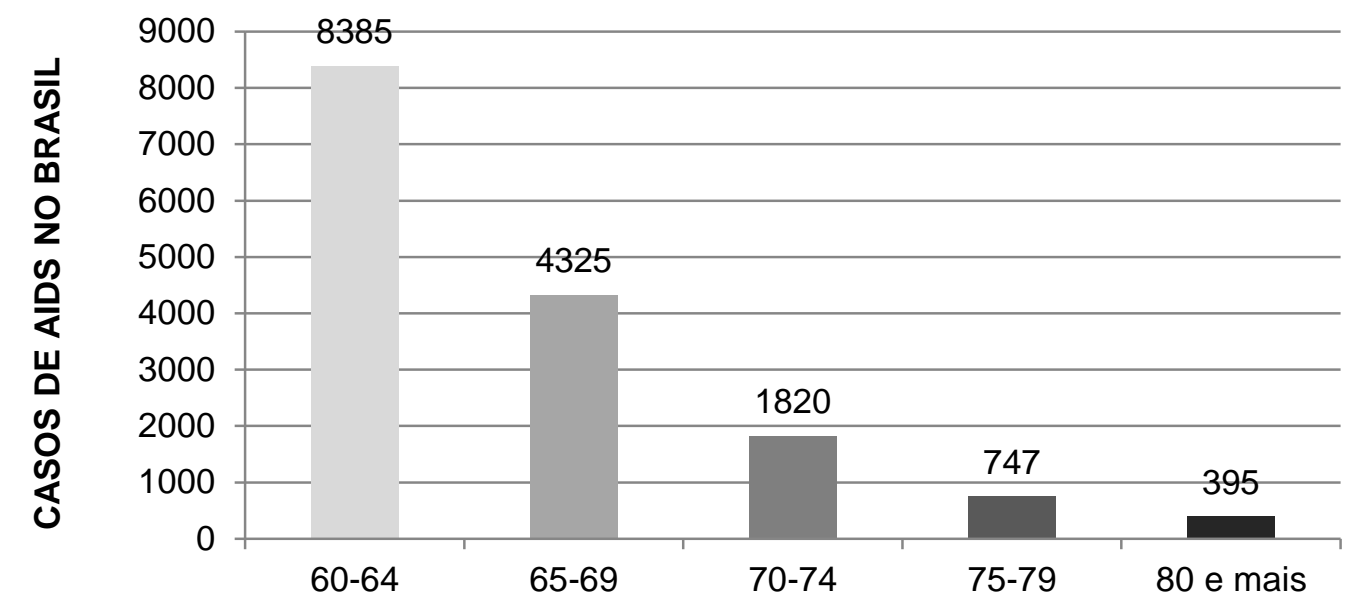

Fonte: Borges JPM, et al., 2021; dados extraídos de TABNET, 2021.

Os primeiros casos de AIDS são datados do ano de 1981 nos Estados Unidos (VERONESI R e FOCACCIA R, 2015). No Brasil, o primeiro caso ocorreu no ano de 1982, mas, apenas em 1985, o MS reconheceu a gravidade do problema para a saúde pública, o que culminou na criação do Programa Nacional da AIDS. Além das políticas públicas nacionais, os movimentos sociais também contribuíram muito para o enfrentamento, prevenção e combate à discriminação as pessoas vivendo com HIV e AIDS (MENDONÇA PME, et al., 2010).

O crescente índice de idosos com HIV/AIDS está relacionado a diversos fatores como: crescimento da população idosa por conta do aumento da expectativa de vida (transição demográfica); permanência da vida sexual mesmo com o envelhecimento devido à melhora da qualidade de vida; avanços na área da saúde, como, por exemplo, o desenvolvimento de medicamentos para disfunções eréteis; invisibilidade do sexo na velhice decorrente do preconceito e falta de informação; baixa adesão e intolerância dos homens idosos aos preservativos masculinos; e inexistência de políticas públicas para prevenção de ISTs direcionadas a este grupo etário (SANTOS AFM e ASSIS M, 2011).

No Brasil, essa problemática do envelhecimento e da AIDS tem conexão direta com preconceito social relacionado ao sexo nesta idade o que demonstra a necessidade de se discutir sobre esse assunto com os idosos e profissionais de saúde que abordam essa população para que eles estejam alertos em relação ao risco de contágio pelo HIV entre os sujeitos com 60 anos e mais, o que pode ocorrer por meio da discussão em grupos de idosos nas Unidades de Saúde da Família (USF) e durante as consultas médicas, além de cursos capacitatórios com os trabalhadores da saúde, a fim de potencializar o conhecimento e as ações de prevenção e tratamento com os idosos (UCHOA YS, et al., 2016).

Os avanços biopsicossociais ocorridos nas últimas décadas e o advento de novas tecnologias para prolongar a vida sexual da população não impediram o estereótipo do idoso assexuado, o qual permanece enraizado na sociedade, influenciando não só as representações dos próprios idosos, como também as políticas públicas que deveriam ser voltadas para esse problema. Além disso, os próprios profissionais de saúde ajudam a reforçar esse preconceito, sobretudo, devido à falta de informação que deveria advir dos programas governamentais de combate as ISTs. Um estudo revelou que $73,1 \%$ dos idosos participantes de grupos da terceira idade já ouviram falar em IST, HIV e AIDS, mas avaliaram que não possuem risco de contrair estas doenças (SANTOS AFM e ASSIS M, 2011). Outro dado sobre a prática do sexo seguro revelou que os idosos relataram conhecer a camisinha, porém, 38\% referiram não saber como utilizá-la corretamente (AGUIAR RB, et al., 2020), demonstrando a necessidade de abordagens e intervenções educativas direcionadas a população idosa. 
Entre os idosos que foram mais notificados com AIDS, estão os indivíduos de 60 até 69 anos. O Instituto Brasileiro de Geografia e Estatística (IBGE) (2018) aponta que essa faixa etária corresponde a 7,5\% da população total, enquanto os idosos acima de 70 e mais são, aproximadamente, $6 \%$. Portanto, diante desses dados, é possível inferir que o maior percentual de acometimento encontrado no presente estudo seja em decorrência da maior proporção de idosos nessa faixa etária (60-69). Outro fator apontado na literatura foi a contaminação pelo HIV ainda na idade adulta (AGUIAR RB, et al., 2020). Estima-se que 50\% das infecções pelo vírus da AIDS nos idosos ocorrem em média aos 56,6 anos (CRUZ GECP, et al., 2020). Além disso, acrescenta-se que a vida sexual entre os idosos até 69 anos é mais ativa em relação aos sujeitos com 70 anos e mais, tendo em vista as alterações fisiológicas e patológicas da senescência (VIEIRA CPB, et al., 2021).

Na tentativa de reduzir o aumento dos casos de HIV e AIDS entre os idosos, foram criados dois projetos pelo governo, em 2008, O Programa Nacional de Doenças Sexualmente Transmissíveis (DST) e AIDS, com finalidade de alertar os sujeitos com 60 anos e mais sobre a importância do uso do preservativo. Em 2009, foi lançada uma nova campanha cujo slogan foi: "Sexo não tem idade para acabar. Proteção também não", a fim de conscientizar acerca dos comportamentos de risco relacionados a infecções sexualmente transmissíveis. Por isso, a Organização Mundial de Saúde (OMS) definiu o Brasil como um dos primeiros países a adotar políticas de HIV/ AIDS voltadas para essa faixa etária (SANTOS AFM e ASSIS M, 2011). Pode-se se conjecturar, que a implantação do programa não foi suficiente para impactar significativamente na redução dos casos, já que houve aumento crescente ao longo dos anos, culminado no pico em 2017, dentre os anos analisados no presente estudo.

Entre todos os casos de AIDS em idosos notificados entre 2009 e 2019, 9,588 eram homens e 6,084 mulheres, havendo uma diferença de $36,5 \%$ se comparados os números de acometidos do sexo masculino e feminino (Gráfico 3). Sendo assim, o número absoluto de homens acometidos foi mais expressivo, quando comparados com as mulheres na mesma faixa etária.

Gráfico 3 - Casos de AIDS identificados no Brasil por ano de notificação no período de 2009 a 2019 de acordo com o sexo na faixa etária de 60 anos e mais.

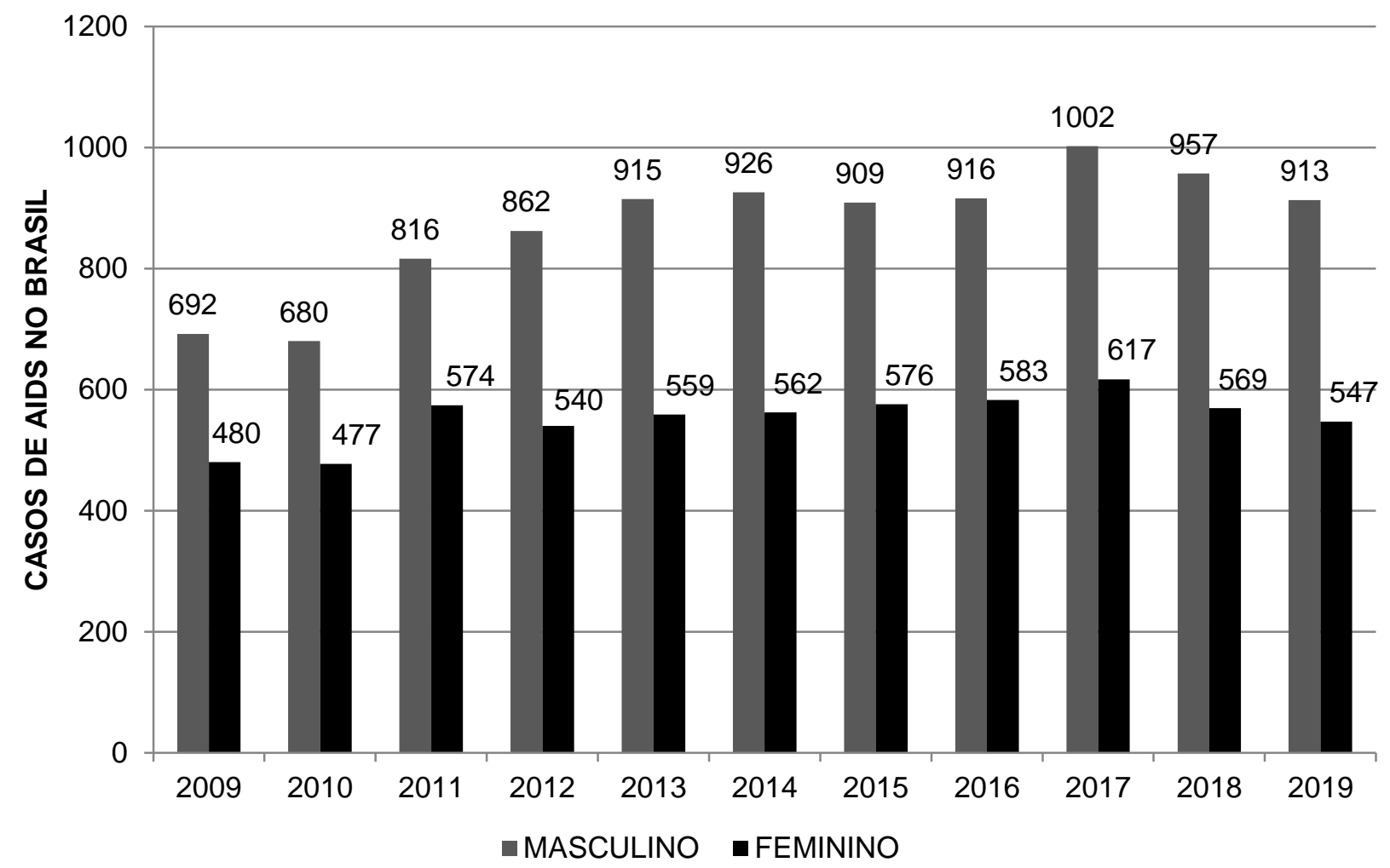

Fonte: Borges JPM, et al., 2021; dados extraídos de TABNET, 2021. 
O gênero dos indivíduos influencia de diversas formas no contágio de HIV e desenvolvimento da AIDS. Em relação às políticas de prevenção da AIDS, as mulheres têm destaque unicamente quando são inseridas em categorias sociais específicas, a exemplo das profissionais do sexo (KNAUTH DR, et al., 2020). Em contrapartida, como fator protetor contra o desenvolvimento das AIDS, estudos demonstraram que mulheres idosas com HIV tem maior conhecimento sobre a sua sexualidade e a doença (AGUIAR RB, et al., 2020).

Em relação ao sexo masculino, a masculinidade hegemônica ainda pregada pela sociedade machista a qual reforça o conceito de que o homem age pelo instinto e possui uma sexualidade desenfreada (MARQUES JUNIOR JS, et al., 2012). Esse fator está relacionado à infidelidade e o relacionamento extraconjugal entre os homens o que também envolve a cultura machista a qual preza pela multiplicidade de parceiros. Além disso, essa cultura ratifica a visão de que as mulheres, principalmente as idosas, devem atuar como mulheres de família que negam o prazer sexual (CERQUEIRA MBR e RODRIGUES RN, 2016). Para mais, existem os mitos em relação à transmissão do vírus entre homossexuais que surgiram junto com os primeiros casos de HIV e permanecem até a atualidade (MARQUES JUNIOR JS, et al., 2012).

Em relação aos óbitos por AIDS em pessoas com 60 anos e mais, foram registradas 12,907 mortes por 100,000 habitantes no período de 2009 até 2019 com uma média por volta de 1,173 casos por ano. Em 2019, foi registrado o maior número de mortes dentre o período estudado, 1,526 por 100,000 habitantes, em contrapartida, no ano de 2009 os registros tiveram menores frequências, 762 por 100,000 habitantes (Gráfico 4). Tais dados revelam o desenvolvimento da AIDS em idosos que já apresentam muitas vezes outras comorbidades associadas, que, conjuntamente, impactam negativamente na evolução do quadro, podendo evoluir para óbito do idoso mesmo diante da possibilidade de tratamento e controle da doença.

Os óbitos também foram estratificados de acordo com o sexo, demonstrando diferenças significativas no número de óbitos por AIDS entre os idosos. Nos anos de 2009 até 2019, foram 8,650 mortes entre indivíduos do sexo masculino e 4,257 do sexo feminino por 100,000 habitantes (Gráfico 4). Nesse interim, 0 número de homens idosos mortos pela AIDS foi, aproximadamente, 50,8\% maior quando comparado aos óbitos entre as mulheres idosas. Assim, é possível inferir que, além de representarem os maiores números de casos de AIDS no período estudado, os homens também morreram mais por essa enfermidade.

Gráfico 4 - Óbitos por AIDS (por 100.000 hab.) no Brasil segundo sexo entre os idosos por ano do óbito no período de 2009 a 2019.

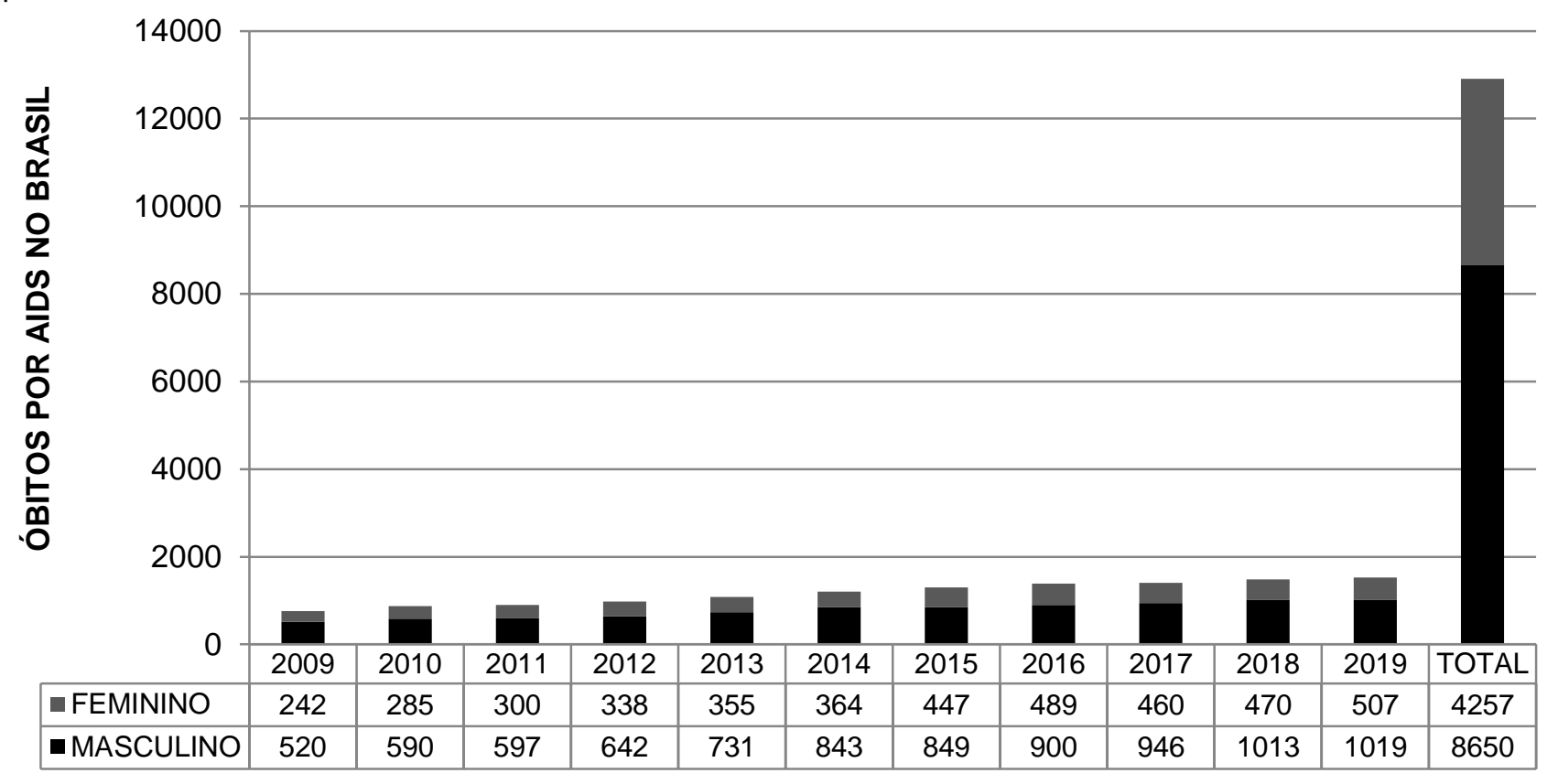

Fonte: Borges JPM, et al., 2021; dados extraídos de Brasil, 2020. 
O vírus HIV se comporta com maior agressividade entre os idosos, alguns estudos demonstram que a idade avançada, além de diminuir a latência entre portar o vírus e apresentar a doença, também reduz a sobrevida diante da doença manifesta (FREITAS EV, et al., 2013). Em pesquisa, idosos diagnosticados tardiamente têm 14 vezes mais chances de morrerem durante o primeiro ano de diagnóstico e 13 vezes mais quando comparados aos que não foram diagnosticados de forma tardia (SMITH RD, et al., 2010).

Além desses fatores, com o envelhecimento, o sistema imune dos idosos é comprometido, o que torna esses indivíduos mais vulneráveis a contaminação pelo HIV, possível desenvolvimento da AIDS e até ao óbito, principalmente, decorrente do diagnóstico tardio. Esse sistema é um processo adaptativo que interage com vários componentes dele mesmo e com o meio ambiente para garantir a integridade do organismo. Com o envelhecimento, ocorre o processo da imunossenescência, caracterizado pela redução na capacidade de resposta do sistema imune diante de uma infecção e também pelo aumento de mediadores inflamatórios circulantes presentes nas doenças crônico-degenerativas. Isso implica o aumento da mortalidade e morbidade da população idosa por doenças infecciosas, bem como por autoimunidade e neoplasias (FREITAS EV, et al., 2013).

A prevenção, desde o início da epidemia, tem sido uma estratégia crucial para os programas de controle da AIDS. No início, era grande o desconhecimento acerca da doença. Desde então, houve um aumento do grau de conhecimento científico acerca do vírus, suas interações com o organismo, sua epidemiologia e sobre os principais determinantes sociais envolvidos. Contudo, ainda hoje, prevenir é uma das formas de combate mais efetivas (AYRES JRCM, 2002).

Nessa conjuntura, a prevenção deve ser ainda mais reforçada e incentivada entre os idosos. O meio mais simples e acessível, independente da faixa etária, é o uso de preservativos masculino e feminino em todas as relações sexuais. Contudo, a disponibilização dos métodos preventivos sem a educação adequada pode acabar sendo menos eficaz, tendo em vista os diversos mitos e preconceitos disseminados sobre a sexualidade, o HIV e a AIDS. Dessa forma, a educação como forma de prevenção deve, também, discutir os diversos fatores comportamentais, culturais e históricos que tornam os indivíduos vulneráveis a infecção pelo HIV (MARQUES JUNIOR JS, et al., 2012).

\section{CONCLUSÃO}

Esse trabalhou possibilitou identificar a prevalência do acometimento dos idosos pela AIDS entre $2009 \mathrm{e}$ 2019 de acordo com o sexo, além dos números de óbitos ocasionados pela enfermidade. Nesse período de tempo, foram notificados 15,672 novos casos de AIDS entre os idosos brasileiros, majoritariamente, na faixa etária entre 60 e 64 anos (8,385), além disso, ocorreram 12,907 óbitos entre os sujeitos com 60 anos e mais. Ademais, constatou-se que os sujeitos do sexo masculino foram mais acometidos em comparação ao sexo feminino em todos os anos analisados. Tendo isso em vista, os dados apontaram para a invisibilidade do problema da AIDS entre os idosos, sendo essencial a educação em saúde dessa população e a maior inclusão dos idosos nos programas governamentais de combate as ISTs.

\section{REFERÊNCIAS}

1. AGUIAR RB, et al. Idosos vivendo com HIV - comportamento e conhecimento sobre sexualidade: revisão integrativa. Ciência \& Saúde Coletiva, 2020; 25(2): 575-584.

2. AYRES JRCM. Práticas educativas e prevenção de HIV/AIDS: lições aprendidas e desafios atuais. Interface Comunicação, Saúde, Educação, 2002; 6(11): 11-24.

3. CERQUEIRA MBR, RODRIGUES RN. Fatores associados à vulnerabilidade de idosos vivendo com HIV/AIDS em Belo Horizonte (MG), Brasil. Ciência \& Saúde Coletiva, 2016; 21(11): 3331-3338.

4. INSTITUTO BRASILEIRO DE GEOGRAFIA E ESTATÍSTICA (IBGE). 2019. Expectativa de vida dos brasileiros aumenta para 76,3 anos em 2018. Disponível em: https://agenciadenoticias.ibge.gov.br/agencia-noticias/2012agencia-de-noticias/noticias/26103-expectativa-de-vida-dos-brasileiros-aumenta-para-76-3-anos-em-2018 Acesso em: 19 jun. 2020.

5. CRUZ GECP, et al. Diagnóstico tardio do Vírus da Imunodeficiência Humana e da Síndrome da Imunodeficiência Adquirida em Idosos: protocolo scoping review. Enfermería Actual de Costa Rica, 2020; (38): 292-299. 
6. FREITAS EV, et al. Tratado de geriatria e gerontologia. 3 ed. Rio de Janeiro: Guanabara Koogan, 2013; 1600p, $1604 p$.

7. GUARNIERI AP. O envelhecimento populacional brasileiro: uma contribuição para o cuidar. Arquivos Brasileiros de Ciências da Saúde, 2008; 33(3): 139-140.

8. INSTITUTO BRASILEIRO DE GEOGRAFIA E ESTATÍSTICA (IBGE). Projeções e estimativas da população do Brasil e das Unidades da Federação. 2018. Disponível em: https://www.ibge.gov.br/apps/populacao/projecao/index.html?utm_source=portal\&utm_medium=popclock\&utm_camp aign=novo_popclock Acessado em: 4 set. 2021

9. KNAUTH DR, et al. O diagnóstico do HIV/aids em homens heterossexuais: a surpresa permanece mesmo após mais de 30 anos de epidemia. Cadernos de Saúde Pública, 2020; 36(6): 1-11.

10. MARQUES JUNIOR JS, et al. Masculinidade hegemônica, vulnerabilidade e prevenção ao HIV/AIDS. Ciência \& Saúde Coletiva, 2012; 17(2): 511-520.

11. MENDONCA PME, et al. Empreendedorismo institucional na emergência do campo de políticas públicas em HIV/AIDS no Brasil. RAE electronica, 2010; 9(1): 1-25.

12. MINISTÉRIO DA SAÚDE. Boletim Epidemiológico de HIV/AIDS. Brasil, 2020. Disponível em: https://www.gov.br/saude/pt-br/assuntos/media/pdf/2020/dezembro/01/boletim-hiv_aids-2020-internet.pdf Acessado em: 18 jun. 2020.

13. SANTOS AFM, ASSIS M. Vulnerabilidade das idosas ao HIV/AIDS: despertar das políticas públicas e profissionais de saúde no contexto da atenção integral: revisão de literatura. Revista Brasileira de Geriatria e Gerontologia, 2011; 14(1): 147-157.

14. SILVA IG, et al. A evolução do HIV/AIDS na terceira idade: uma revisão bibliográfica. Biblioteca virtual de enfermagem - Cofen. 2015. Disponível em: http://biblioteca.cofen.gov.br/wp-content/uploads/2015/06/A-evolucao-doHIV-AIDS-na-terceira-idade-uma-revisao-bibliografica.pdf. Acesso em: 4 set. 2021.

15. SMITH RD, et al. HIV transmission and high rates of late diagnoses among adults aged 50 years and over. AIDS, 2010; 24(13): 2109-2115.

16. SOUSA LRM, et al. Representações sociais do HIV/AIDS por idosos e a interface com a prevenção. Revista Brasileira de Enfermagem, 2019; 72(5): 1129-1136.

17. TRAVASSOS GF, et al. The elderly in Brazil: demographic transition, profile, and socioeconomic condition. Revista Brasileira de Estudos de População, 2020; 37: 1-27.

18. UCHOA YS, et al. A sexualidade sob o olhar da pessoa idosa. Rev. bras. geriatr. gerontol., 2016.

19. VERONESI R, FOCACCIA R. Tratado de Infectologia. 5 ed. São Paulo: Atheneu, 2016. 2 v; $165 p$.

20. VIEIRA CPB, et al. Tendência de infecções por HIV/Aids: aspectos da ocorrência em idosos entre 2008 e 2018. Escola Anna Nery, 2021; 25(2): 1-8. 